\title{
Mucosal Immunization with a pH-Responsive Nanoparticle Vaccine Induces Protective CD8+ Lung-Resident Memory T Cells
}

\author{
Frances C. Knight, Pavlo Gilchuk, Amrendra Kumar, Kyle W. Becker, \\ Sema Sevimli, Max E. Jacobson, Naveenchandra Suryadevara, \\ Lihong Wang-Bishop, Kelli L. Boyd, James E. Crowe, Jr., \\ Sebastian Joyce, and John T. Wilson
}

${ }^{1}$ Department of Biomedical Engineering, Vanderbilt University, Nashville, TN 37235, USA

${ }^{2}$ Department of Pathology, Microbiology, and Immunology, Vanderbilt University Medical Center,

Nashville, TN 37232, USA

${ }^{3}$ Vanderbilt Vaccine Center, Vanderbilt University Medical Center, Nashville, TN 37232, USA

${ }^{4}$ Department of Veterans Affairs Tennessee Valley Healthcare System, Nashville, TN 37212, USA

${ }^{5}$ Department of Chemical and Biomolecular Engineering, Vanderbilt University, Nashville, TN 37235, USA

${ }^{6}$ Vanderbilt Institute for Infection, Immunology, and Inflammation, Vanderbilt University Medical Center, Nashville, TN 37232, USA

${ }^{7}$ Department of Pediatrics, Vanderbilt University Medical Center, Nashville, TN 37232, USA

${ }^{8}$ Chemical and Physical Biology Program, Vanderbilt University, Nashville, TN 37235, USA

${ }^{9}$ Department of Cell and Developmental Biology, Vanderbilt University, Nashville, TN 37235, USA

${ }^{10}$ Vanderbilt Center for Immunobiology, Vanderbilt University Medical Center, Nashville, TN 37232, USA

${ }^{11}$ Vanderbilt-Ingram Cancer Center, Vanderbilt University Medical Center, Nashville, TN 37232, USA

*Corresponding Author: john.t.wilson@vanderbilt.edu 
A)

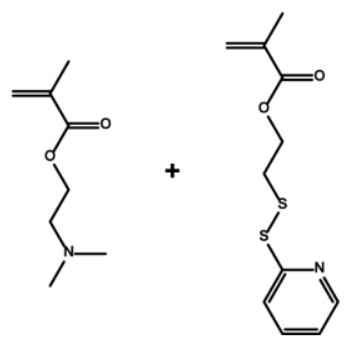

DMAEMA (92\%)

PDSMA (8\%)

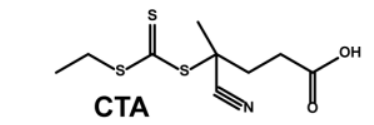

100:1 [M] $]_{0}:[\mathrm{CTA}]_{0}$ $20: 1[\mathrm{CTA}]_{0}:[\mathrm{I}]_{0}$ $40 \mathrm{wt} \%$ in dioxane $30{ }^{\circ} \mathrm{C}, 18 \mathrm{~h}$

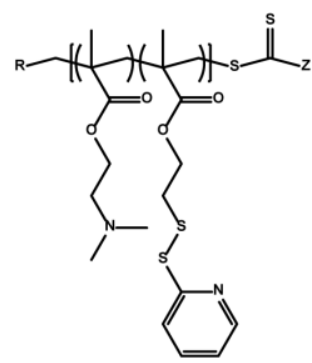

DMAEMA-co-PDSMA macroCTA (mCTA)

B)

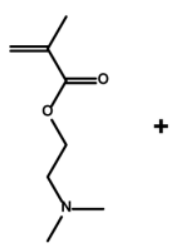<smiles>C=C(C)C(=O)CCCCC</smiles><smiles>C=C(C=O)C(=O)CCC</smiles>

DMAEMA (30\%) BMA $(40 \%)$

PAA $(30 \%)$

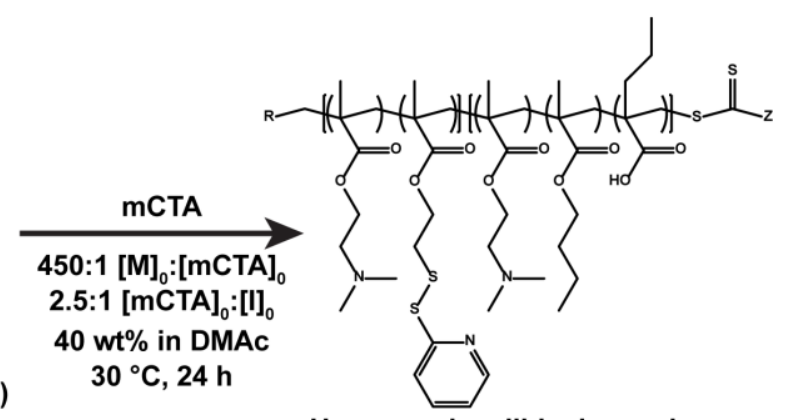

pH-responsive diblock copolymer

C)

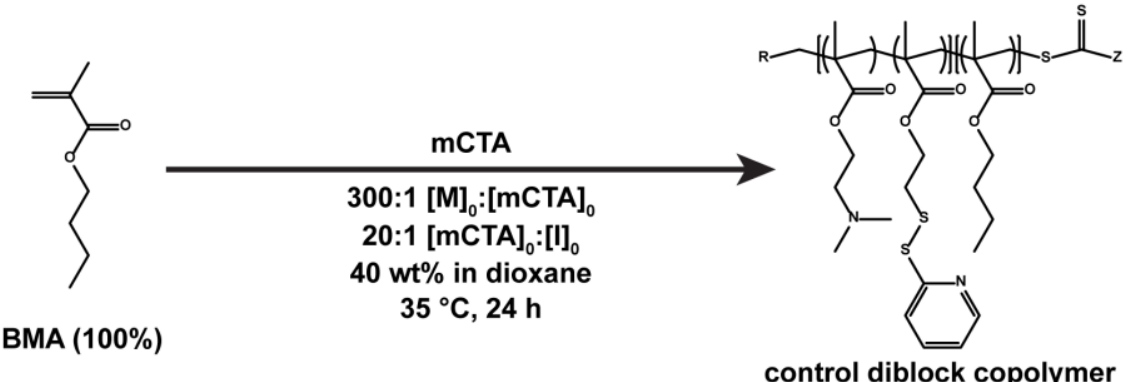

Scheme S1. RAFT synthesis of pH-responsive and control polymers for dualdelivery of protein antigen and nucleic acid adjuvant-related to Methods. (A) Synthesis scheme and reaction conditions for DMAEMA-co-PDSMA macro-chain transfer agent (mCTA). (B) Synthesis of pH-responsive diblock copolymer, (DMAEMAco-PDSMA)-b-(PAA-co-DMAEMA-co-BMA). (C) Synthesis of control diblock copolymer, (DMAEMA-Co-PDSMA)- $b$-BMA.

Table S1-related to Methods. Summary of polymer properties.

\begin{tabular}{|l|c|c|c|c|c|c|}
\hline & \multicolumn{3}{|c|}{ pH-responsive polymer } & \multicolumn{3}{c|}{ Control polymer } \\
\hline & $\begin{array}{c}\mathbf{1}^{\text {st }} \text { block } \\
\text { (mCTA) }\end{array}$ & $\begin{array}{c}\mathbf{2}^{\text {nd }} \\
\text { block }\end{array}$ & $\begin{array}{c}\text { Diblock } \\
\text { copolymer (1+2) }\end{array}$ & $\begin{array}{c}\mathbf{1}^{\text {st }} \text { block } \\
\text { (mCTA) }\end{array}$ & $\begin{array}{c}\mathbf{2}^{\text {nd }} \\
\text { block }\end{array}$ & $\begin{array}{c}\text { Diblock } \\
\text { copolymer (1+2) }\end{array}$ \\
\hline $\begin{array}{l}\text { Molecular } \\
\text { weight (g/mol) }\end{array}$ & 11,370 & 18,470 & 29,840 & 12,870 & 29,600 & 42,470 \\
\hline $\begin{array}{l}\text { Polydispersity } \\
\text { index (PDI) }\end{array}$ & 1.04 & - & 1.51 & 1.04 & - & 1.09 \\
\hline
\end{tabular}



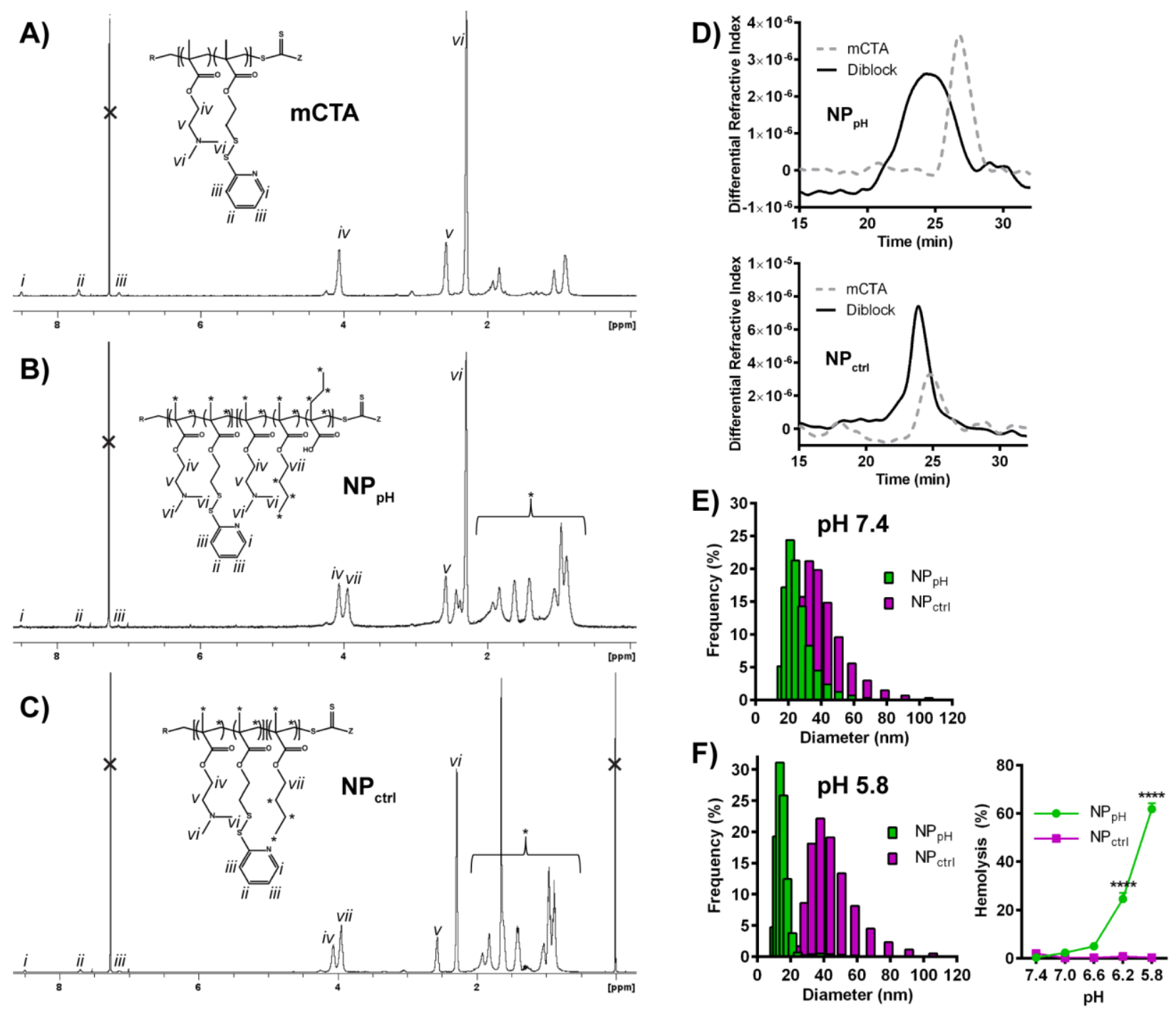

Figure S1. Polymer and nanoparticle characterization for $\mathrm{pH}$-responsive and control polymers-related to Methods. (A-C) Representative ${ }^{1} \mathrm{H}-\mathrm{NMR}\left(\mathrm{CDCl}_{3}\right)$ of (A) DMAEMA-co-PDSMA macroCTA, (B) pH-responsive diblock copolymer, and (C) control diblock copolymer. (D) Representative GPC traces for mCTA and diblock copolymer, pH-responsive (top) and control (bottom). (E) Representative size distribution (number average) at $\mathrm{pH} 7.4$ for $\mathrm{pH}$-responsive $\mathrm{NP}\left(\mathrm{NP}_{\mathrm{pH}}\right)$ and control $\mathrm{NP}\left(\mathrm{NP}_{\mathrm{ctr}}\right)$ as measured by DLS. (F) Left: representative size distribution (number average) at $\mathrm{pH} 5.8$ for $\mathrm{NP}_{\mathrm{pH}}$ and $\mathrm{NP}_{\mathrm{ctrl}}$, indicating $\mathrm{pH}$-dependent change in particle morphology; right: erythrocyte lysis assay demonstrates $\mathrm{pH}$-dependent membrane destabilizing activity of $\mathrm{NP}_{\mathrm{pH}}$ but not $\mathrm{NP}_{\text {ctrl }}(10 \mu \mathrm{g} / \mathrm{ml}$ polymer $)$. Data are mean \pm SEM with $n=4$ per group. ${ }^{* \star * \star} p<0.0001$ by ordinary two-way ANOVA with Sidak's multiple comparisons test. 

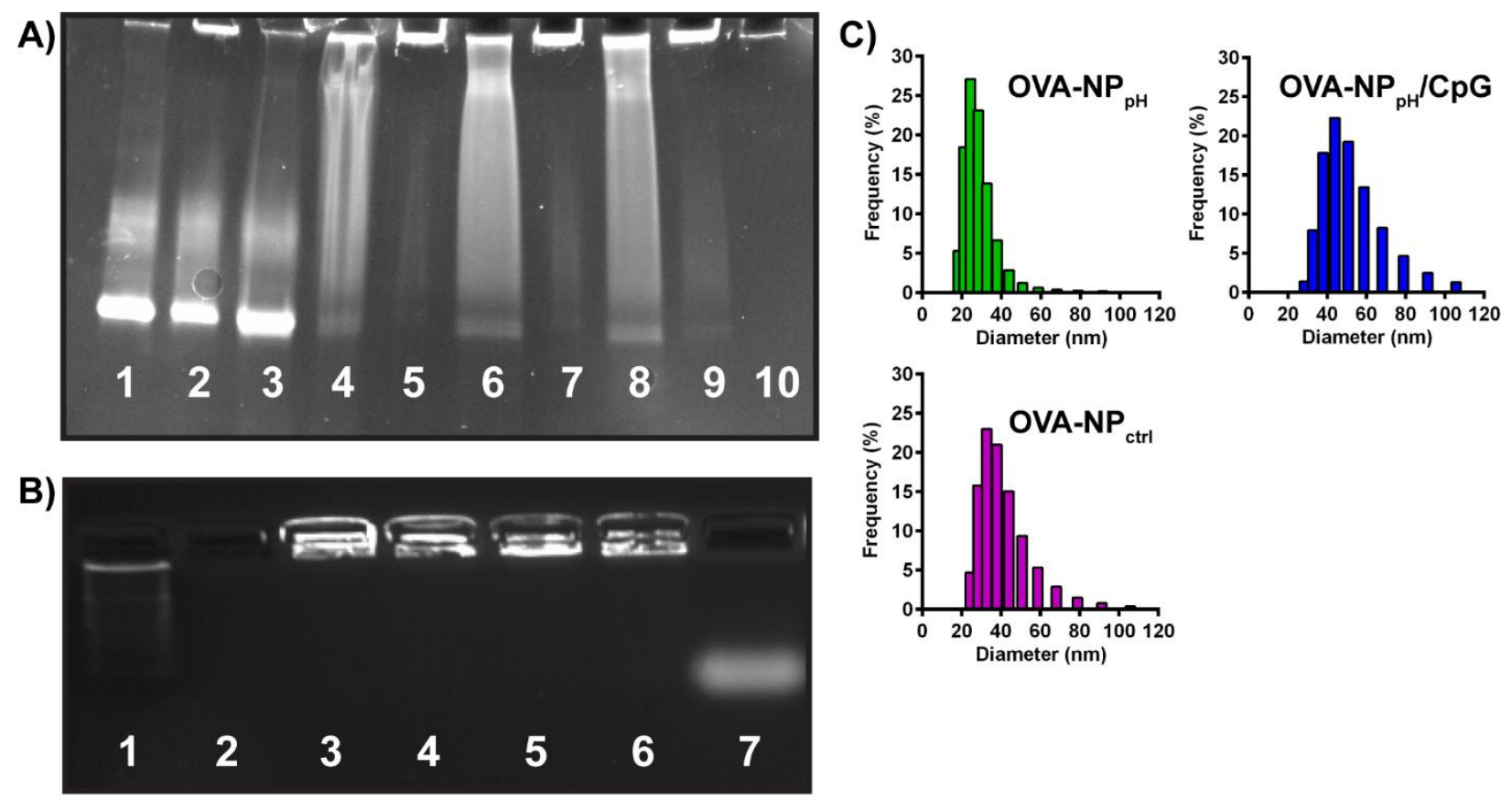

Figure S2. OVA-nanoparticle conjugation and adjuvant complexation-related to Methods. (A) Thiolated OVA protein labeled with FITC was reacted with NP made from $\mathrm{pH}$-responsive polymer $\left(\mathrm{NP}_{\mathrm{pH}}\right)$ or control polymer $\left(\mathrm{NP}_{\mathrm{ctrl}}\right)$ to form conjugates at various molar ratios of OVA:polymer. SDS-PAGE was used to confirm antigen conjugation. Lane (1) free OVA protein; (2) mixture of OVA+NP $c$ ctr; (3) mixture of $O V A+N P_{p H}$; (4) OVA-NPpH (1:20 molar ratio); (5) OVA-NP ctrl $_{1}$ (1:14); (6) OVA-NP pH $_{\text {(1:10); (7) OVA-NP }}$ crl $(1: 7)$;

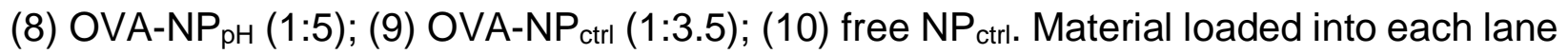
was normalized to $5 \mu \mathrm{g}$ OVA. Thiolated OVA was confirmed to conjugate to both $\mathrm{NP}_{\mathrm{pH}}$ and $\mathrm{NP}_{\text {ctrl }}$ at all molar ratios tested (lanes 4-9), and non-thiolated OVA did not conjugate with either polymer (lanes 2-3). The NP itself was not fluorescent (lane 10). (B) CpG DNA was complexed with nanoparticles $\left(\mathrm{NP}_{\mathrm{pH}}\right)$ and conjugates $\left(\mathrm{OVA}-\mathrm{NP}_{\mathrm{pH}}, 1: 5\right)$ at various charge ratios of polymer:CpG (+/-). Gel electrophoresis and GelRed staining were used to confirm adjuvant complexation. Lane (1) 20 bp ladder; (2) OVA-NP; (3) NP/CpG (6:1 +/-); (4) NP/CpG (4:1); (5) OVA-NP/CpG (6:1); (6) OVA-NP/CpG (4:1); (7) free CpG. Material loaded into each lane was normalized to $2.3 \mu \mathrm{g} \mathrm{CpG}$. CpG complexed with both NP and OVA-NP at both charge ratios, as shown by lack of migration from the wells of the gel (lanes 3-6). Free CpG migrated from the well due to its net negative charge (lane 7). OVA-NP did not show background staining from GelRed (lane 2). (C) Representative size distributions (number average) at pH 7.4 for OVA-NP $\mathrm{pH}_{\text {(1:5 molar }}$ ratio), OVA-NP ${ }_{\text {ctrl }}$ (1:3.5 molar ratio), and $\mathrm{OVA}-\mathrm{NP}_{\mathrm{pH}} / \mathrm{CpG}$ (1:5 molar ratio, 6:1 charge ratio), as measured by DLS. 

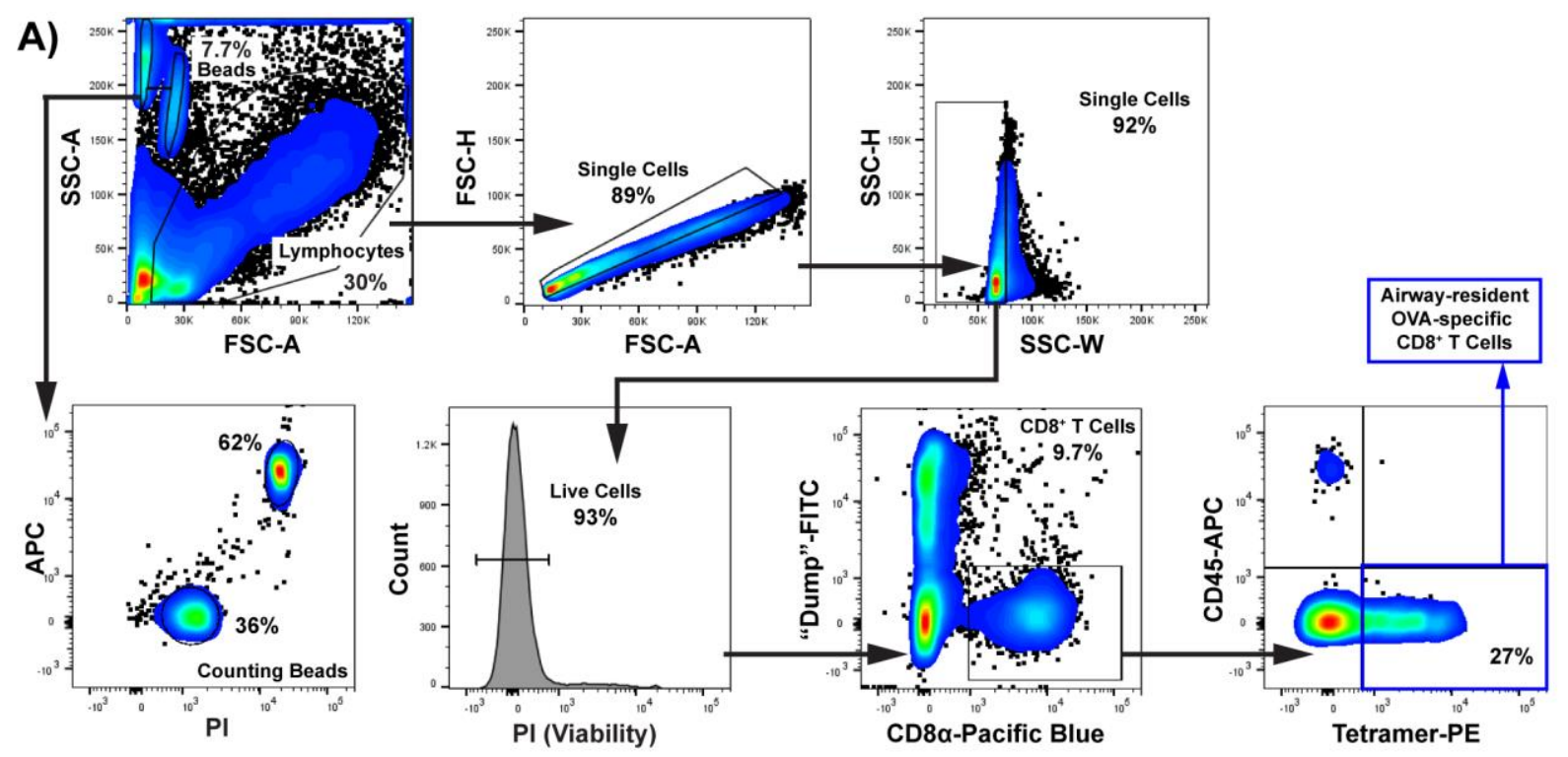

B) Gated on single cells

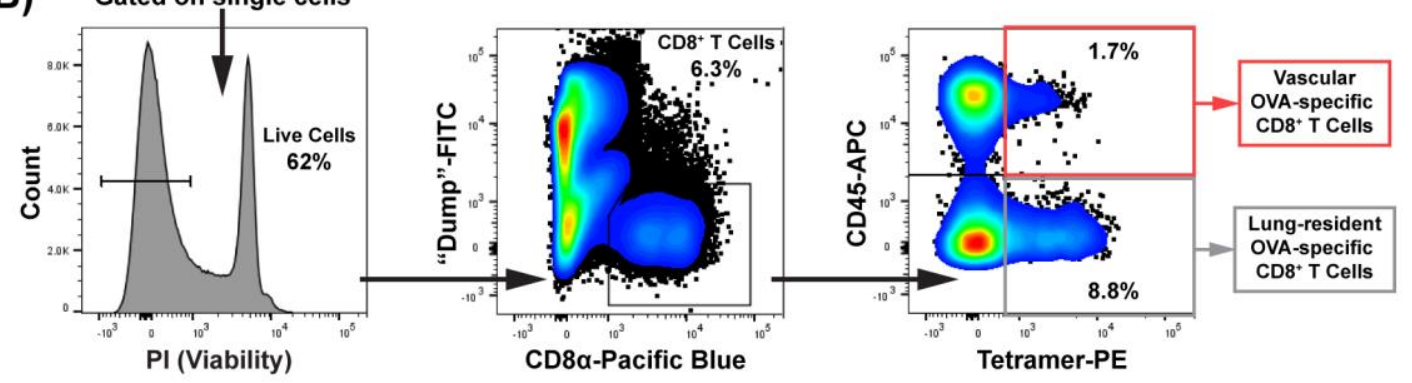

C) Gated on single cells
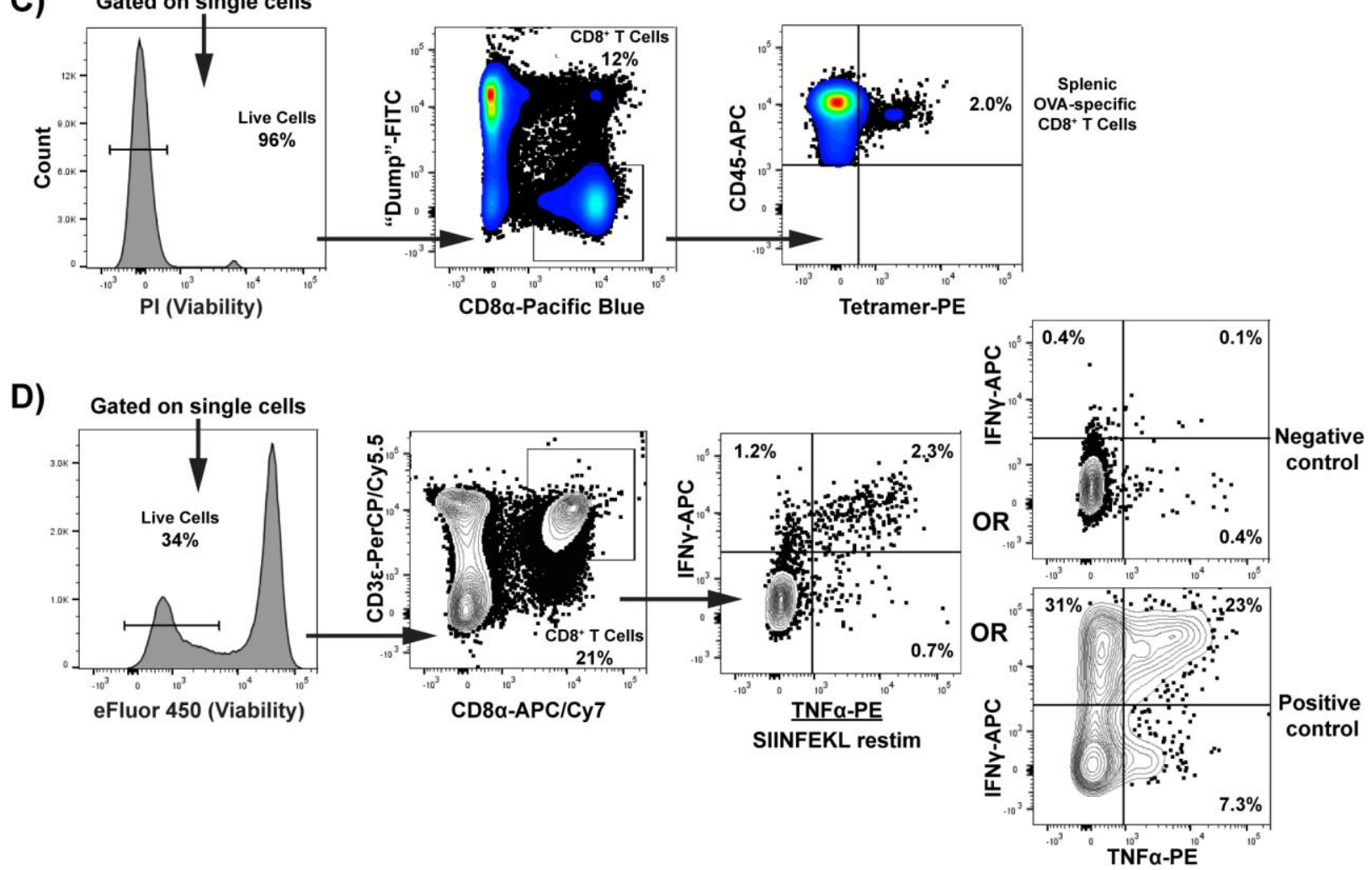
Figure S3. Gating strategies for flow cytometric analysis of antigen-specific CD8 ${ }^{+}$ $T$ cells and intracellular cytokine production in airways, lungs (interstitium and vasculature), and/or spleens-related to Figures 1-4, 7, 9. Representative plots are from mice immunized with OVA-NP $\mathrm{pH}_{\mathrm{H}} / \mathrm{CpG}$. (A-C) Events were first gated on lymphocytes (SSC-A vs. FSC-A), then single cells were isolated via gating FSC-H vs. FSC-A and SSC$\mathrm{H}$ vs. SSC-W. Single cells negative for propidium iodide (PI) were live cells. Viable CD8 ${ }^{+}$ $\mathrm{T}$ cells were positive for CD8 $\alpha$-Pacific Blue and negative for the "dump" channel (B220/CD4/CD11b/CD11c-FITC). Two populations of counting beads were gated for calculating absolute cell counts. (A) Analysis of airway (AW)-resident OVA-specific CD8 ${ }^{+}$ T cells in BAL. AW population was negative for i.v. stain (CD45-APC) and positive for PE-labeled SIINFEKL/MHC-I tetramer (tetramer-PE+). (B) Analysis of lung interstitium (IST)-resident and marginated vascular (MV) OVA-specific CD8 ${ }^{+} \mathrm{T}$ cells in lungs. IST population was $\mathrm{CD}^{2} 5-\mathrm{APC}^{-}$and tetramer-PE${ }^{+}$. MV population was $\mathrm{CD}^{+} 5^{+}$tetramer ${ }^{+}$. (C) Analysis of systemic OVA-specific $\mathrm{CD}^{+} \mathrm{T}$ cells in spleens. Spleen population was tetramer-PE+ after being gated on CD8a. (D) For ICCS, counting beads, "dump" channel, and i.v. staining were not used. After gating for lymphocytes and singlets, viability was determined using eFlour 450, and viable CD8 ${ }^{+} T$ cells were CD3 $\varepsilon^{+} C D 8 \alpha^{+}$. Cells were then gated on TNFa-PE and IFNY-APC to assess cytokine production. Plots shown here are from a lung sample; the same gating was used for spleens. BAL was not collected for ICCS experiments. Cells were restimulated with SIINFEKL peptide, CRPMI (negative control), or PMA/ionomycin (positive control). 
A)

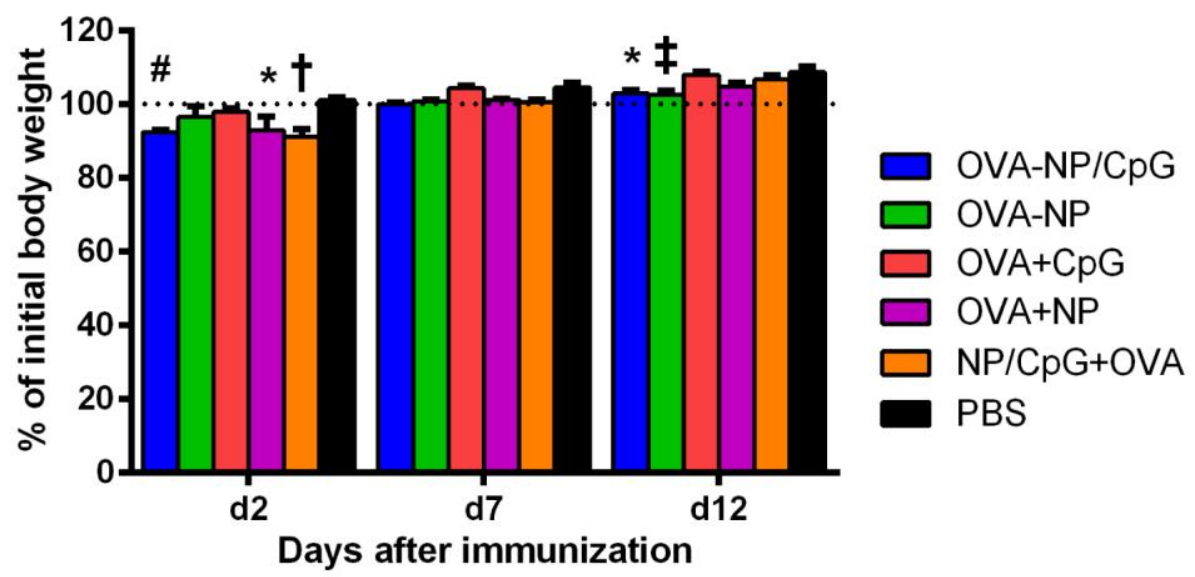

B) OVA-NP/CpG OVA+CpG

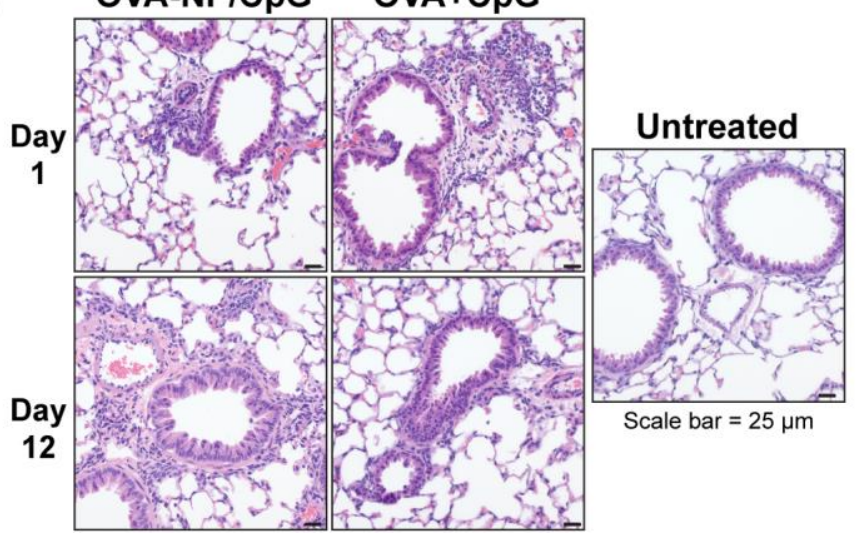

Figure S4. Minimal toxicity after pulmonary immunization with pH-responsive formulations-related to Figure 3. (A) Weight loss shown as percent of body weight on day of immunization (d0). Dose: $25 \mu \mathrm{g} \mathrm{NP}, 7.5 \mu \mathrm{g}$ OVA, $1.4 \mu \mathrm{g} \mathrm{CpG}$. Mice were immunized with $\mathrm{pH}$-responsive formulations: OVA-NP/CpG, OVA-NP, OVA+CpG, $\mathrm{OVA}+\mathrm{NP}, \mathrm{NP} / \mathrm{CpG}+\mathrm{OVA}$, or PBS (control). Data are mean $\pm \mathrm{SEM}$ and pooled from five independent experiments, with $n=2-18$ per group. ${ }^{*} p<0.05, \pm p<0.01,+p<0.001$, $\# p<0.0001$, by ordinary two-way ANOVA with Tukey multiple comparisons test. Significance is indicated relative to the PBS control group. All unlabeled groups are ns (not significant). 
A)
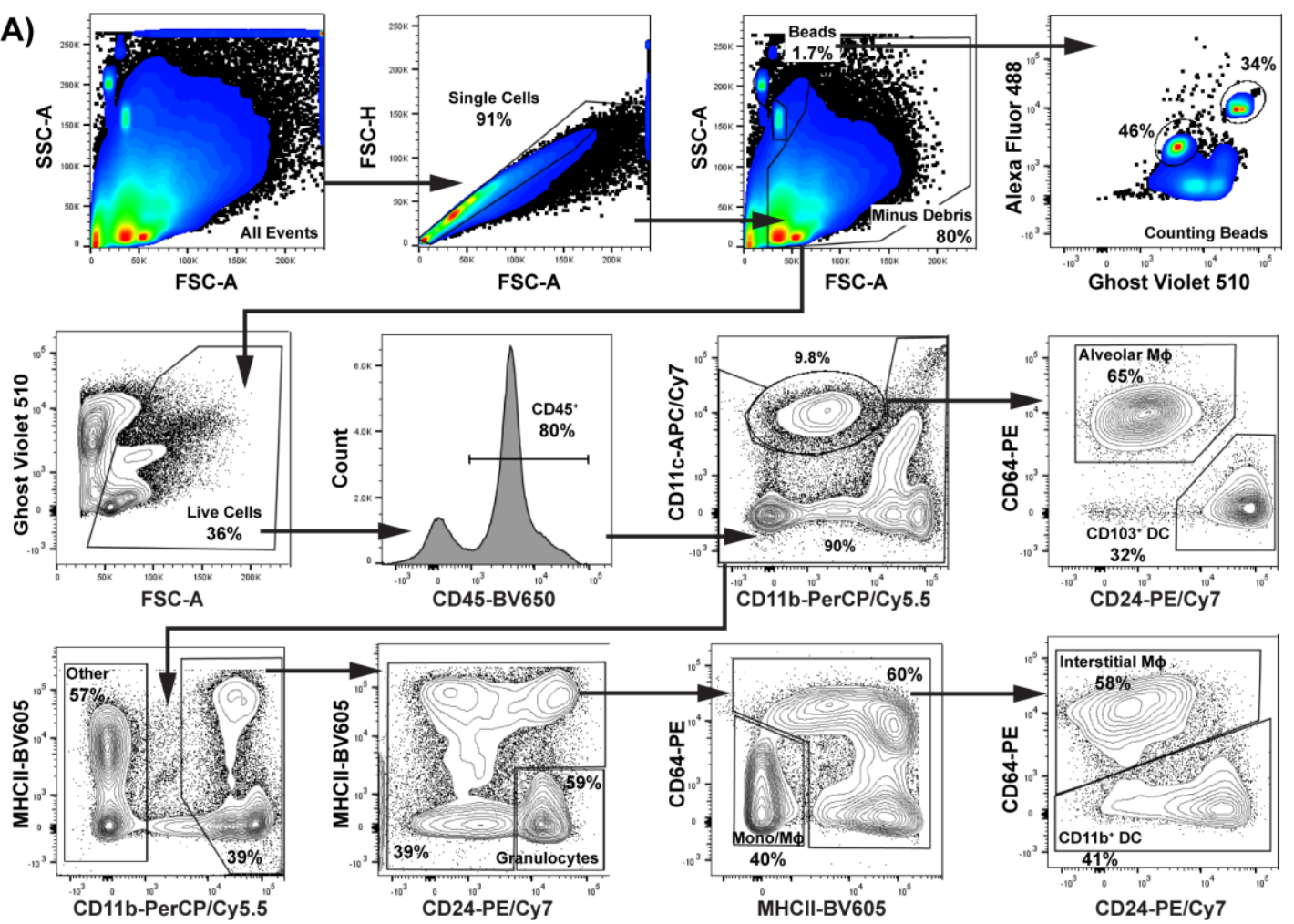

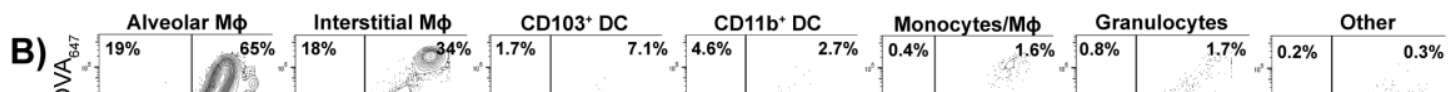

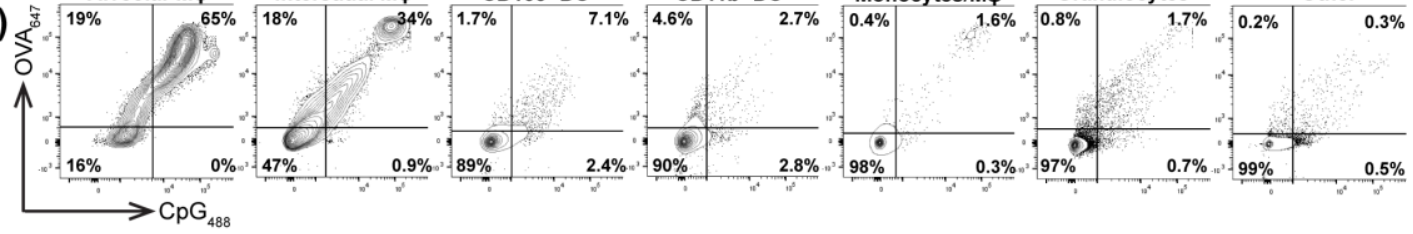

C)

OVA MFI $\times$ Count: DCs CpG MFI $\times$ Count: DCs

$24 \mathrm{~h}$

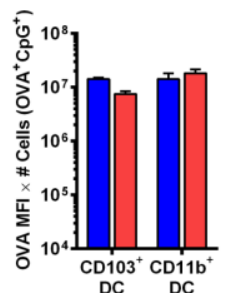

$48 \mathrm{~h}$
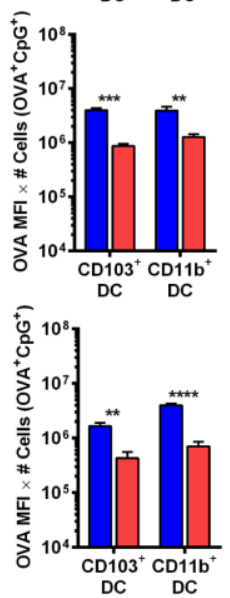
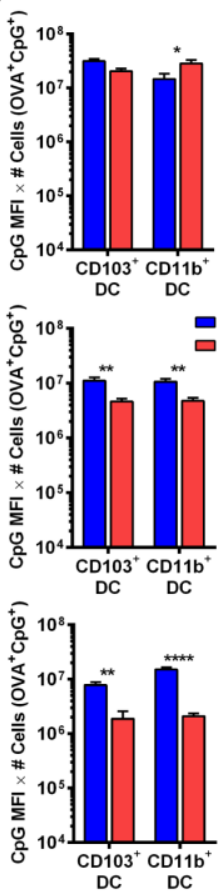

D)

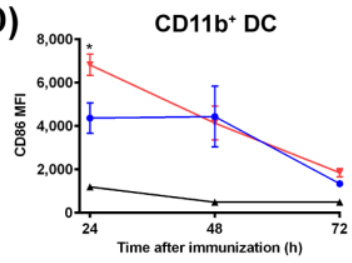

Alveolar $\mathrm{M} \varphi$
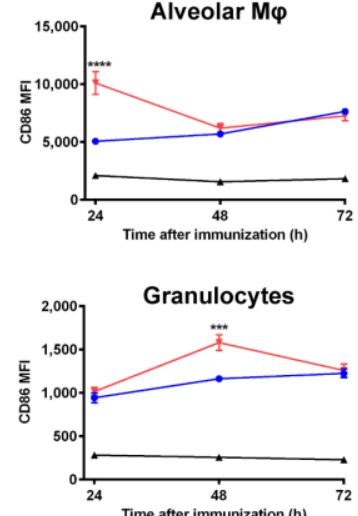

- $\mathrm{OVA}_{547}-\mathrm{NP}^{2} \mathrm{CPG}_{488}$

$+\mathrm{OVA}_{547}+\mathrm{CPG}_{488}$ 
Figure S5. Gating strategy for flow cytometric analysis of $\mathrm{OVA}_{647}$ and $\mathrm{CpG}_{488}$ in pulmonary innate immune cells, representative dot plots showing uptake in pulmonary APCs, relative OVA and CpG uptake in DC subsets, and CD86 expression in selected subsets-related to Figure 5. The gating strategy shown here was adapted from Misharin et al. ${ }^{56}$ Flow plots are from mice immunized with $\mathrm{pH}$ responsive $\mathrm{OVA}_{647}-\mathrm{NP} / \mathrm{CpG} \mathrm{G}_{48}$. (A) All events were gated on single cells (FSC-H vs. FSCA), then debris was gated out (SSC-A vs. FSC-A). Two populations of counting beads were gated for use in calculating absolute cell counts. Viable single cells were gated using Ghost Dye ${ }^{\mathrm{TM}}$ Violet 510 . Viable $\mathrm{CD} 45^{+}$cells were then gated using a variety of surface markers to identify pulmonary immune cell subsets. (B) Representative dot plots showing $\mathrm{OVA}_{647}$ vs. $\mathrm{CpG}_{488}$ uptake for each cell type. Double-positive cells $\left(\mathrm{OVA}^{+} \mathrm{CpG}^{+}\right)$were indicative of vaccine cargo co-localization. (C) Relative OVA and CpG uptake in CD103+ $\mathrm{DC}$ and $\mathrm{CD} 11 \mathrm{~b}^{+} \mathrm{DC}$ for each formulation was calculated as OVA MFI $\times \# \mathrm{OVA}^{+} \mathrm{CpG}^{+}$ cells and $\mathrm{CpG} \mathrm{MFI} \times \# \mathrm{OVA}^{+} \mathrm{CpG}^{+}$cells. (D) CD86 MFI in $\mathrm{CD}^{2} 1 \mathrm{~b}^{+} \mathrm{DCs}$, alveolar macrophages, and granulocytes. Data are mean \pm SEM with $n=3-4$ per group. ${ }^{*} p<0.05$, ${ }^{* *} p<0.01,{ }^{* * *} p<0.001,{ }^{* * *} p<0.0001$ by (C) ordinary two-way ANOVA with Sidak's multiple comparisons test, or (D) ordinary two-way ANOVA comparing OVA-NP/CPG vs. OVA+CpG, with Tukey multiple comparisons test. 


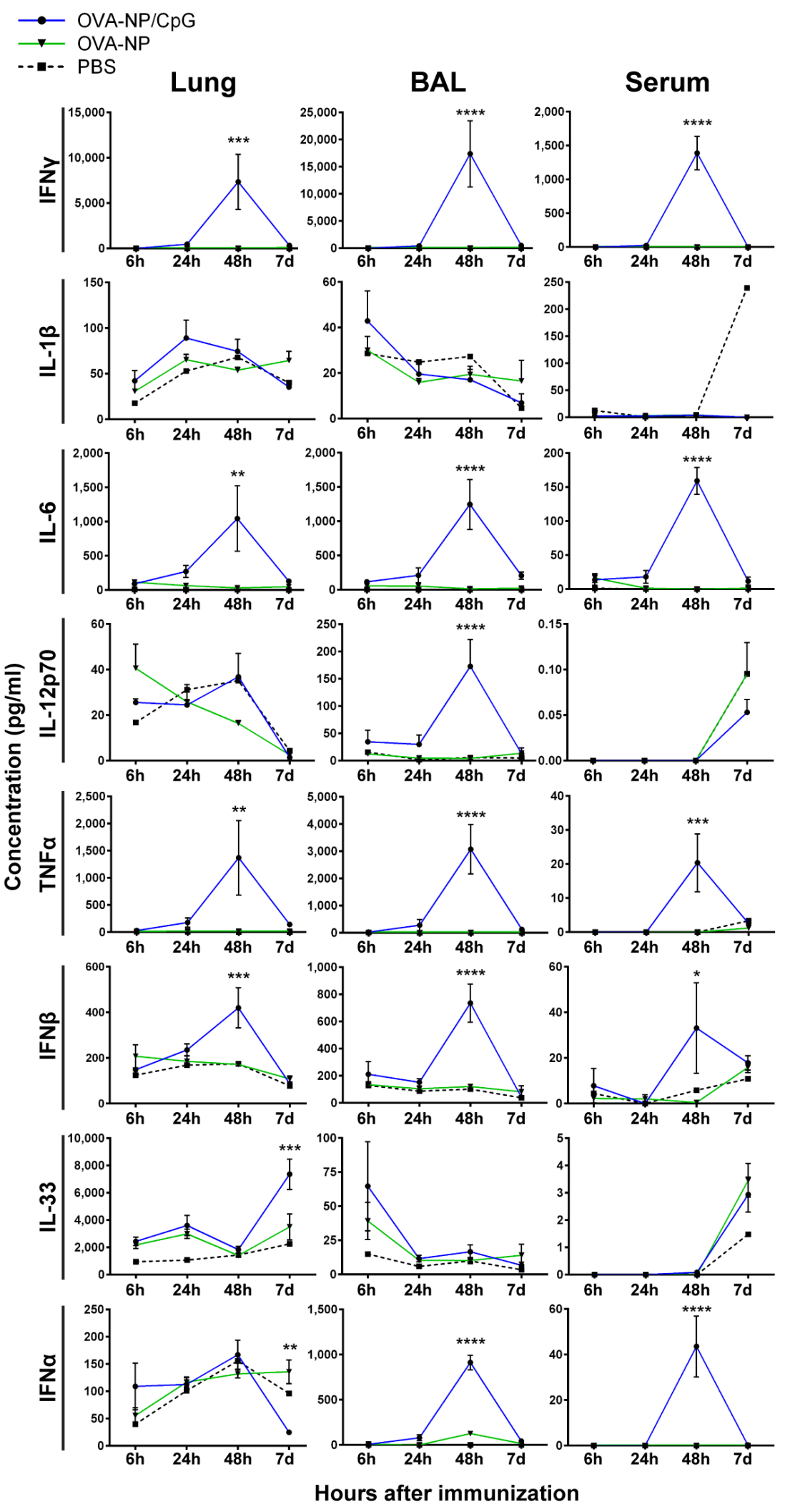


Figure S6. Acute cytokine response to nanoparticle vaccine is localized, transient, and supportive of lung-resident $\mathrm{CD8}^{+} \mathrm{T}$ cells - related to Figure 6. Cytokines associated with CD8 ${ }^{+} \mathrm{T}$ cells (IFNy, IL-1 $3, \mathrm{IL}-6, \mathrm{IL}-12 \mathrm{p} 70$ ) and $\mathrm{T}_{\mathrm{RM}}$ generation (TNF $\alpha$, IFN $\beta$, IL-33, IFNa) were measured in lungs, BAL, and serum obtained $6 \mathrm{~h}, 24 \mathrm{~h}, 48 \mathrm{~h}$, or $7 \mathrm{~d}$ after immunization with either OVA-NP/CpG or OVA-NP. Data are mean \pm SEM and representative of two independent experiments, with $n=4-5$ per group. Immunization dose: $25 \mu \mathrm{g} \mathrm{NP}, 7.5 \mu \mathrm{g}$ OVA, $1.4 \mu \mathrm{g} \mathrm{CpG} .{ }^{*} p<0.05,{ }^{* *} p<0.01,{ }^{* * *} p<0.001,{ }^{* * *} p<0.0001$, by ordinary two-way ANOVA with Tukey's multiple comparisons test. Statistical differences shown are for comparison of OVA-NP/CpG vs. OVA-NP. 

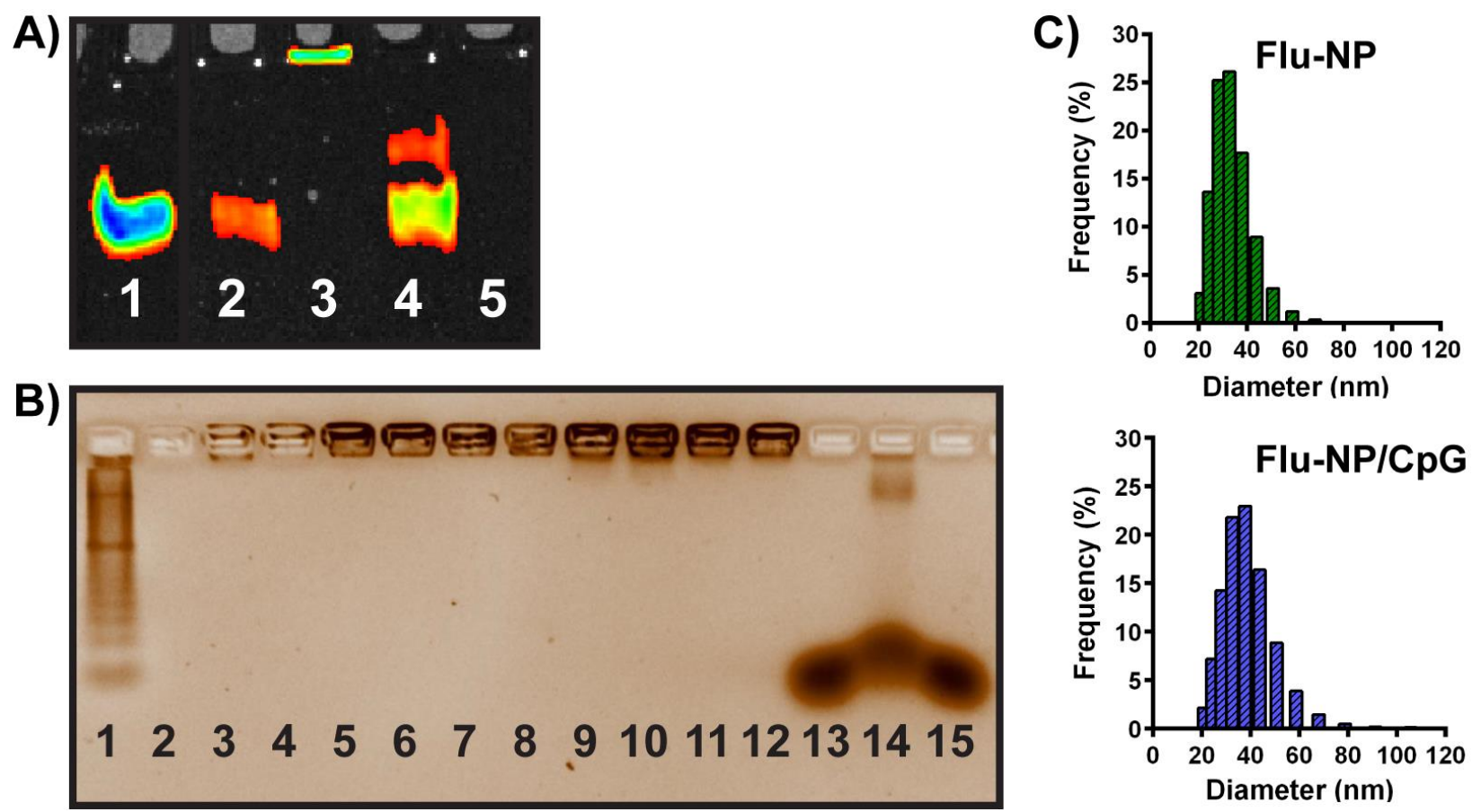

Figure S7. Flu-nanoparticle conjugation and adjuvant complexation-related to Methods. (A) Thiolated Flu protein labeled with AF647 was reacted with NP made from $\mathrm{pH}$-responsive polymer at a molar ratio of 5:1 (protein:polymer) to form conjugates. SDSPAGE was used to confirm antigen conjugation. Lane (1) free Flu protein; (2) thiolated Flu protein; (3) Flu-NP conjugate; (4) Flu-NP conjugate + TCEP; (5) free NP. Material loaded into each lane was normalized to $3.5 \mu \mathrm{g}$ Flu protein. Thiolated Flu was confirmed to conjugate to NP (lane 3), as incubation with TCEP to disrupt the disulfide bridges between thiolated protein and NP resulted in reappearance of the free protein band (lane 4, compare with lane 1). Thiolating the protein did not cause significant self-aggregation (lane 2). The NP itself was not fluorescent (lane 5). (B) CpG DNA was complexed with nanoparticles (NP) and conjugates (OVA-NP or Flu-NP, 1:5) at various charge ratios of polymer:CpG (+/-). Gel electrophoresis and GelRed staining were used to confirm adjuvant complexation. Lane (1) 20 bp ladder; (2) OVA-NP; (3-4) Flu-NP; (5) NP/CpG (6:1); (6) NP/CpG (4:1); (7) OVA-NP/CpG (6:1); (8) OVA-NP/CpG (4:1); (9) Flu-NP/CpG (6:1); (10) Flu-NP/CpG (4:1); (11) Flu-NP/CpG (6:1); (12) Flu-NP/CpG (4:1); (13) OVA+CpG; (14) Flu+CpG; (15) free CpG. CpG complexed with NP, OVA-NP, and Flu-NP at both charge ratios, as shown by lack of migration from the wells of the gel (lanes 5-12). Free $\mathrm{CpG}$ and $\mathrm{CpG}$ mixed with OVA or Flu protein migrated from the well due to its net negative charge (lanes 13-15), indicating the NP is necessary for complete electrostatic complexation. Antigen-NP conjugates did not show background staining from GelRed (lanes 2-4). (C) Representative size distributions (number average) at $\mathrm{pH}$ 7.4 for Flu-NP (1:5 molar ratio) and Flu-NP/CpG (1:5 molar ratio, 6:1 charge ratio), as measured by DLS. 